TABEA SCHIMMÖLLER, Vechta

\title{
Wie verstehen Schülerinnen und Schüler den Begriff der Un- endlichkeit?
}

Dass für die Mathematik und die Analysis im Besonderen die Unendlichkeit von zentraler Bedeutung ist, scheint unverkennbar und so bezeichnete WeYL (1966) die Mathematik sogar als „Wissenschaft von der Unendlichkeit". Doch obwohl der Unendlichkeit in der Mathematik eine besondere Rolle zukommt, taucht sie im Unterrichtsgeschehen der Sekundarstufe I selten bzw. gar nicht auf und wird weder in den Bildungsstandards noch in dem Kerncurriculum des Fachs Mathematik explizit erwähnt. Dennoch werden mathematische Inhalte thematisiert und damit Anforderungen an die Schülerinnen und Schüler gestellt, die zwingend Unendlichkeitsaspekte beinhalten, wie bspw. die Erläuterung der Zahlbereichserweiterung, das Darstellen von Zahlen auf der Zahlengeraden oder das Rechnen mit unendlich periodischen Dezimalbrüchen (vgl. NK, 2006). Wie verstehen also Schülerinnen und Schüler den Begriff der Unendlichkeit, der nicht direkt behandelt wird, aber dennoch implizit eine große Rolle spielt? Und inwieweit sind ihnen die verschiedene Unendlichkeitsaspekte, denen sie im Laufe ihrer Schulzeit im Fach Mathematik begegnen, vertraut?

\section{Forschungsstand}

In wichtigen einschlägigen Arbeiten zum Unendlichkeitsbegriff (PIETZSCH, 1967; PiageT \& INHELDER, 1971; TALL, 1977; Arbeitsgruppe um FISCHBEIN, 1979; vgl. dazu BEUTELSPACHER \& WeIGAND, 2002; WinTER, 2000, MARX, 2006; TSAMIR \& TIROSH, 2006) wird deutlich, dass der Unendlichkeitsbegriff durch seine Vielschichtigkeit ein sehr schwer zu fassender Ausdruck ist und die Vorstellungen der Probanden über die Unendlichkeit auffallend defizitär sind. Jedoch muss an dieser Stelle auch kritisch angemerkt werden, dass insgesamt nur wenige Studien existieren, die sich mit dem Phänomen der Unendlichkeit auseinandersetzen und dass viele dieser Untersuchungen auch wenig aktuell sind. Zudem konzentriert sich der Großteil der Arbeiten auf die Begegnung mit der Unendlichkeit in der Sekundarstufe II bzw. im Studium. Die Schülerinnen und Schüler werden aber schon sehr viel früher mit der Unendlichkeit konfrontiert.

\section{Untersuchungsdesign}

Um zu ermitteln, was Schülerinnen und Schüler unter Unendlichkeit verstehen und was sie damit verbinden, wurde auf eine empirische Untersuchung zurückgegriffen, welche sich aus den vier Phasen Standortbestimmung 1, pädagogische Intervention, Standortbestimmung 2 und Follow- 
Up-Test zusammensetzte. Als Probanden wurden Schülerinnen und Schüler der 8. Jahrgangsstufe der Realschule gewählt, die sowohl mit den natürlichen, ganzen und rationalen Zahlen vertraut sind und zudem bereits mit unendlich periodischen Dezimalbrüchen umgehen mussten. Eruiert werden soll unter anderem, welches individuelle Verständnis die Schülerinnen und Schüler von Unendlichkeit, von der Mächtigkeit der Zahlbereiche, von unendlich periodischen Dezimalbrüchen und von der Dichtheit der rationalen Zahlen haben. Zur Untersuchung wurden insgesamt vier Gruppen ausgewählt, welche wiederum in die zwei Untersuchungsstränge Experimentalund Kontrollgruppe unterteilt wurden, sodass die Erhebung an insgesamt zwei Realschulen in jeweils zwei achten Klassen des gleichen Jahrganges erfolgte. Zur Standortbestimmung wurde ein Fragebogen ausgearbeitet, um das Verständnis und die Vorstellungen der Schülerinnen und Schüler vom Unendlichkeitsbegriff zu erheben. Zwischen Standorterhebung 1 und 2 wurde eine pädagogische Intervention durchgeführt, wobei der experimentelle Stimulus mit einer Unterrichtseinheit gesetzt wurde, welche für eine Dauer von fünf Unterrichtsstunden konzipiert wurde und als Thematik „Gleichmächtigkeit von Mengen“ beinhaltete. Dieser Unterrichtsinhalt wurde gewählt um zu eruieren, inwieweit die Schülerinnen und Schüler in der Lage sind, einen völlig neuen Unendlichkeitsaspekt aufzunehmen und inwieweit die Auseinandersetzung mit der Unendlichkeit die Gedanken der Probanden in Gang setzt. Zwei Monate nach der pädagogischen Intervention folgte ein Follow-Up-Test mit der Durchführung von Einzelinterviews, um die Ansichten einzelner Probanden zur Unendlichkeit näher zu ergründen und um die Nachhaltigkeit der Intervention zu untersuchen.

\section{Exemplarischer Ausschnitt aus dem Datenmaterial}

Erste Ergebnisse der Untersuchung liegen noch nicht vor, daher soll mit der Auswahl von vier Fragen aus dem Fragebogen ein erster Einblick in das Datenmaterial gegeben.

\section{Schüler: PASCAL}

(1) Was verstehst du unter ,unendlich“?

$>$ "Ich verstehe darunter, dass wenn man 1000 Billion nimmt, die Zahl danach."

(2) Nenne ein Beispiel, wo etwas Unendliches vorkommt.

$>$,Weiß ich nicht.“

(3) Wo findest du in der Mathematik etwas Unendliches?

$>$,bei der Periode, da geht es immer weiter z.B. 0,333333 $\overline{3}$.“

(4) Wie viele Bruchzahlen gibt es zwischen $\frac{1}{3}$ und $\frac{2}{3}$ ? Begründe deine Antwort.

$>$,Ich glaube dazwischen gibt es keine Zahlen weil es die direkte Zahl danach ist." 


\section{$\underline{\text { Schülerin: MARIE }}$}

(1) Was verstehst du unter ,unendlich“?

$>$ „Unendlich ist wenn etwas immer weitergeht, es zwar einen Anfang hat aber wie im Wort beschrieben kein Ende hat. Etwas Unendliches muss aber nicht unbedingt einen Anfang haben."

(2) Nenne ein Beispiel, wo etwas Unendliches vorkommt.

$>$ „Das Universum, es wird vermutet, dass es unendlich ist, denn es ist noch nicht genau erforscht."

(3) Wo findest du in der Mathematik etwas Unendliches?

$>$,z.B. eine Gerade. Sie hat keinen Anfang oder ein Ende so wie etwa eine Strecke."

(4) Wie viele Bruchzahlen gibt es zwischen $\frac{1}{3}$ und $\frac{2}{3}$ ? Begründe deine Antwort.

, $\frac{1}{3}, \frac{1,1}{3}, \frac{1,2}{3}, \frac{1,3}{3}, \frac{1,4}{3}, \frac{1,5}{3}, \frac{1,6}{3}, \frac{1,7}{3}, \frac{1,8}{3}, \frac{1,9}{3}, \frac{2}{3}$ Also 10 Stück.“

\section{Schülerin: SONJA}

(1) Was verstehst du unter ,unendlich“?

„Unendlich bedeutet für mich, dass etwas sehr lange sich fortsetzt wie bei z.B. $0, \overline{3}$, da geht die 3 unendlich weiter.“

(2) Nenne ein Beispiel, wo etwas Unendliches vorkommt.

$>$,Ein Ameisenhaufen mit ganz vielen Ameisen. Da weiß man nicht wie viele drin sind. Also unendlich.“

(3) Wo findest du in der Mathematik etwas Unendliches?

„Bei z. B. $0, \overline{4}$ da geht die 4 ja unendlich weiter

(4) Wie viele Bruchzahlen gibt es zwischen $\frac{1}{3}$ und $\frac{2}{3}$ ? Begründe deine Antwort.

$>$ „Es gibt dort unendlich viele Zahlen. Da man die Zahlen immer erweitern kann."

\section{Schüler: VICTOR}

(1) Was verstehst du unter ,unendlich“?

„Dass es kein Ende gibt, irgendwann fängt alles von vorne an wie so ein Kreis.“

(2) Nenne ein Beispiel, wo etwas Unendliches vorkommt.

$>$,Donut, Pizza also die Form hat weder Anfang noch Ende“

(3) Wo findest du in der Mathematik etwas Unendliches?

$>$ „Ein Kreis, eine 8 und $\infty$ und Periode.“

(4) Wie viele Bruchzahlen gibt es zwischen $\frac{1}{3}$ und $\frac{2}{3}$ ? Begründe deine Antwort.

$>$,Viele, Begründung $\frac{2}{4}, \frac{1}{2}$ usw.“

Pascal und auch Sonja scheinen mit der Unendlichkeit etwas zu verbinden, das für sie nicht mehr beschreibbar ist, so bspw. eine Zahl die sie nicht mehr benennen können oder die Anzahl von Ameisen in einem Ameisen- 
haufen. Marie hingegen beschreibt die Unendlichkeit als etwas ohne Anfang und Ende und ist in der Lage den Begriff sowohl zu definieren, abzugrenzen als auch mit geometrischen Beispielen zu untermauern. Victor wiederum verbindet das Unendliche mit einem Kreislauf und stützt dieses durch das Beispiel eines Kreises. Der Unendlichkeitsaspekt, dass zwischen zwei rationalen Zahlen unendlich viele weitere rationale Zahlen existieren, scheint anhand dieses exemplarischen Auszugs nur Sonja bekannt zu sein. Da sie zuvor jedoch Ameisen in einem Ameisenhaufen als ein Beispiel für etwas Unendliches nennt, muss noch weiteres Datenmaterial herangezogen werden, um genauere Aussagen über ihr Verständnis treffen zu können.

\section{Ausblick}

Anhand dieses nur kleinen Auszugs wird deutlich, wie breit gefächert und individuell die Vorstellungen der Schülerinnen und Schüler über Unendlichkeit sind. Zudem lässt sich erkennen, dass das Verstehen dieses Begriffs und dass das Wissen über Unendlichkeit keinesfalls selbstverständliches Nebenprodukt des Umgangs mit Mathematik sind. Der Begriff der Unendlichkeit, als fundamentaler und faszinierender Begriff in der Mathematik, ist durch die Verankerung in so vielen mathematischen Inhalten auch im Mathematikunterricht ständig präsent und durch eine Vernachlässigung bei der Begriffsvermittlung können Defizite entstehen, welches sich auf das Verstehen von Zusammenhängen auswirken.

\section{Literatur}

Beutelspacher, A. \& Weigand, H.-G. (2002): Endlich...unendlich! in: Mathematik lehren, 112, S. 4-8.

Marx, A. (2006): Schülervorstellungen zu „unendlichen Prozessen“. Texte zur mathematischen Forschung und Lehre 50. Hildesheim, Berlin: Franzbecker Verlag.

Niedersächsisches Kultusministerium (NK) (2006): Kerncurriculum für die Realschule. Schuljahrgänge 5-10. Hannover: Unidruck.

Tsamir, P. \& Tirosh, D. (2006): PME 1 to 30 - Summing up and looking ahead: A personal perspective on infinitive sets, in: Novotná, J., Moraová, H., Krátká, M. \& Stehlíková, N (Hg.): Proceedings of the 30th Conference of the International group for the Psychology of Mathematics Education, Vol. 1, Prague, S. 49-63.

Weyl, H. (1966): Philosophie der Mathematik und Naturwissenschaft, 3. Aufl., München: R. Oldenbourg.

Winter, M. (2000): Am liebsten habe ich nur gerechnet... . Reflexionen zu Einstellungen von Lehramtsstudenten zur Mathematik und zum Mathematikunterricht, in: Institut für Didaktik der Naturwissenschaften, der Mathematik und des Sachunterrichts (Hrsg.): Zugänge zur Mathematik: Lernprozesse - Verfahren - Einstellungen, Vechtaer fachdidaktische Forschungen und Berichte, Heft 2, S. 35 - 58. 